\title{
Correction: Honey Bee Inhibitory Signaling Is Tuned to Threat Severity and Can Act as a Colony Alarm Signal
}

\section{The PLOS Biology Staff}

\section{Notice of Republication}

This article was republished on May $19^{\text {th }}, 2016$ to correct poor figure quality in the PDF version, which was introduced during the typesetting process. The publisher apologizes for these issues with the figure quality. Please download this article again to view the updated version.

\section{Reference}

1. Tan K, Dong S, Li X, Liu X, Wang C, Li J, et al. (2016) Honey Bee Inhibitory Signaling Is Tuned to Threat Severity and Can Act as a Colony Alarm Signal. PLoS Biol 14(3): e1002423. doi:10.1371/journal.pbio. 1002423 PMID: 27014876

\section{G OPEN ACCESS}

Citation: The PLOS Biology Staff (2016) Correction: Honey Bee Inhibitory Signaling Is Tuned to Threat Severity and Can Act as a Colony Alarm Signal. PLoS Biol 14(6): e1002496. doi:10.1371/journal. pbio. 1002496

Published: June 9, 2016

Copyright: ๑ 2016 The PLOS Biology Staff. This is an open access article distributed under the terms of the Creative Commons Attribution License, which permits unrestricted use, distribution, and reproduction in any medium, provided the original author and source are credited. 